\author{
2006 ERSD Annual Report \\ DOE-BER Environmental Remediation Sciences Project \#1020499
}

\title{
Biomolecular Mechanisms Controlling Metal and Radionuclide Transformations in Anaeromyxobacter dehalogenans
}

\author{
PIs: Alexander S. Beliaev and James K. Fredrickson \\ Co_PIs: Frank E. Löffler and Robert A. Sanford \\ Collaborators: Matthew J. Marshall, Grigoriy E. Pinchuk \\ Pacific Northwest National Laboratory, Richland, WA
}

\section{Research Objectives:}

Microbiological reduction and immobilization of U(VI) and Tc(VII) has been proposed as a strategy for remediating radionuclide-contaminated environments. Numerous studies focusing on the reduction kinetics and speciation of these metals have been carried out using contaminated sediment samples, microbial consortia, and pure bacterial cultures. While previous work with model organisms has increased the general understanding of radionuclide transformation processes, fundamental questions regarding radionuclide reduction mechanisms by indigenous microorganisms are poorly understood, especially under the commonly encountered scenario where multiple electron acceptors are present. Therefore, the overall goal of the proposed research is to elucidate the molecular mechanisms of radionuclide biotransformation by Anaeromyxobacter dehalogenans, a predominant member of indigenous microorganism commonly found in contaminated subsurface environments, and to assess the effects of relevant environmental factors affecting these transformation reactions. The following specific scientific questions are being addressed:

- What are the mechanisms of metal and radionuclide reduction in organisms indigenous to contaminated FRC sediments? Specifically, what genes and pathways contributes to $\mathrm{Fe}(\mathrm{III}), \mathrm{U}(\mathrm{VI})$ and $\mathrm{Tc}(\mathrm{VII})$ reduction in A. dehalogenans 2CP-C? Are membraneassociated high-molecular weight $c$-type cytochromes involved in metal/radionuclide reduction in this organism?

- How are genes involved in metal/radionuclide reduction in A. dehalogenans strain 2CP-C regulated under different redox conditions? What are the global gene expression patterns in A. dehalogenans 2CP-C under Fe(III), U(VI), Tc(VII), nitrate-, and 2-chlorophenolreducing conditions?

- What are the key environmental factors specific to FRC subsurface environments that affect the expression of $A$. dehalogenans genes involved in metal/radionuclide reduction? In particular, how do low $\mathrm{pH}$ and the presence of co-contaminants (such as nitrate and chlorinated compounds) impact the gene expression and radionuclide transformation rates in A. dehalogenans?

The research integrates targeted physiological and genetic analyses with microarray expression and genotype profiling studies to elucidate the mechanisms of metal transformation reactions in an environmentally relevant bacterial group. This research effort will expand our knowledge of the mechanisms involved in metal reduction, and enhance our predictive capability of the processes that govern radionuclide transformation reactions in subsurface environments. Ultimately, these findings will assist the design and implementation of more efficient 
bioremediation approaches to enhance the reductive transformation and immobilization of radionuclides at contaminated DOE sites.

\section{Research Progress and Implications:}

During the first year of the project, we focused a significant portion of our efforts on development of a system for genetic manipulations to defining genome-encoded properties of $A$. dehalogenans 2CP-C contributing to the metal reduction potential of the organism. As of October 2006, we have adopted a number approaches for introducing random (transposon-based) and targeted mutations in 2CP-C that will allow us to identify genes/proteins involved in respiration of metal and radionuclide electron acceptors. The pools of mutants deficient in genes encoding multiheme $c$-type cytochromes as well as other redox active proteins are being screened for the ability to transfer electrons to both solid and complexed forms of Fe(III) as well as soluble $\mathrm{U}(\mathrm{VI})$ and $\mathrm{Tc}(\mathrm{VII})$.

We have also designed whole-genome oligonucleotide microarrays for high-throughput expression studies using the Affymetrix Gene-Chip platform. The fabricated arrays contain 200,000 probes that represent 4,433 open reading frames and 4,567 intergenic sequences and cover $99 \%$ of the $A$. dehalogenans 2CP-C genome. The first set of expression profiling experiments using Fe(III)-, nitrate-, and fumarate-grown batch cultures of 2CP-C are in progress to establish the base-line transcriptome patterns of the organism under various respiratory conditions.

We have also initiated experiments to establish optimal growth parameters using controlled cultivation (chemostats). The optimization of the cultivation conditions, in particular during steady-state growth under defined conditions is critical for achieving the level of detail and precision required to delineate the role various environmental factors on the metal and radionuclide abilities of $A$. dehalogenans 2CP-C. The experiments are performed under controlled conditions with respect to growth rate, electron donor and acceptor concentrations, and relevant parameters such as dissolved oxygen, temperature and $\mathrm{pH}$. Upon achieving steady state growth conditions, the cells from continuous cultures will be transitioned to electron donorlimiting and non-limiting conditions using various concentrations of metal-electron acceptors.

In resting cell reduction assays, Anaeromyxobacter strains 2CP-C and 2CP-1 rapidly

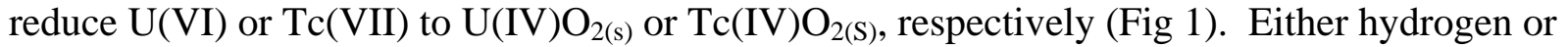
acetate can be used in this process as electron donors. The sub-cellular localization of reduced $\mathrm{UO}_{2}$ or $\mathrm{TcO}_{2}$ nanoparticles is both periplasmic and extracellular, which is very similar to what is seen in Shewanella (suggestive that the reduction pathways may be similar). Initial kinetic studies to ascertain the rates of radionuclide reduction relative to $S$. oneidensis MR-1 have been completed. Interestingly, strains 2CP-C and 2CP-1 displayed significant differences in the reduction rates of $\mathrm{U}(\mathrm{VI})$ or $\mathrm{Tc}(\mathrm{VII})$. Strain $2 \mathrm{CP}-1$ reduces $\mathrm{U}(\mathrm{VI})$ and $\mathrm{Tc}(\mathrm{VII})$ at much faster rates than either strain 2CP-C or MR-1. However, the differences in the sub-cellular localization of the $\mathrm{UO}_{2}$ or $\mathrm{TcO}_{2}$ nanoparticles were indistinguishable between Anaeromyxobacter strains. Xray Absorption Fine Structure (XAFS) studies demonstrated that the $\mathrm{UO}_{2}$ and $\mathrm{TcO}_{2}$ nanoparticles were chemically similar to biogenic $\mathrm{UO}_{2}$ and $\mathrm{TcO}_{2}$ standards produced by Shewanella, however, average nanoparticle size was slightly larger than reported for Shewanella (data not shown).

As shown by our previous work with S. oneidensis MR-1 (Marshall et al. (2006) PLoS Biology 4:1324-1333), c-type cytochromes are important mediators of electron transfer to radionuclides. The genome of $A$. dehalogenans $2 \mathrm{CP}-\mathrm{C}$ (the only strain currently sequenced) encodes over 40 multiheme $c$-type cytochromes; some containing more than 20 putative $c$-type 
heme binding domains (Fig 2). The function of these high molecular weight cytochromes, which possess an atypically large numbers of heme molecules per protein, is currently unknown. To study the involvement of these unusual cytochromes in radionuclide reduction, we developed a genetic system for targeted and random mutagenesis. Using both approaches; we are currently conducting functional studies to characterize the electron transport network of 2CP-C involved in radionuclide reduction. This information will subsequently be used for comparative studies, after the genomes of 3 other Anaeromyxobacter strains become available. We are working with JGI on sequencing strains 2CP-1, strain K, and strain FW-109. The latter was isolated from the FRC site.

Preliminary studies indicate that both A. dehalogenans 2CP-C and 2CP-1 localized $\mathrm{UO}_{2}$ nanoparticles with an exopolymeric substance (EPS) similar to Shewanella (Fig. 1 and Marshall et al.). In collaboration with another ERSP-funded project which is focused on identifying the biogeochemical mechanisms controlling reduced radionuclide particle properties and stability, we have begun to study the process of EPS association with reduced metal oxides produced by Anaeromyxobacter. We are currently investigating the factors influencing the $\mathrm{UO}_{2}$-EPS matrix production by Anaeromyxobacter. Studies are also underway to determine if the EPS matrix exhibits physical properties homologous to the heme-protein-containing glycocalyx excreted by Shewanella. The sub-cellular localization of $\mathrm{TcO}_{2}$ nanoparticles produced by Anaeromyxobacter was also determined to be similar to $\mathrm{TcO}_{2}$ nanoparticle localization by Shewanella, however; neither organism localized $\mathrm{TcO}_{2}$ nanoparticles with an EPS matrix. This finding suggests that the radionuclide reduction pathways containing multiple hydrogenases and other electron transfer proteins that may also constitute integral parts of the terminal reductase complexes for radionuclide biotransformation. These findings are anticipated to have important implications for understanding biotransformation, immobilization, and long-term fate of biogenic $\mathrm{UO}_{2}$ and $\mathrm{TcO}_{2}$ in subsurface environments.

\section{Planned Activities:}

Research during the next year of this 3-year project will focus on:

- Identification of key components of the (metal) respiratory chain of A. dehalogenans 2CP-C and extend the finding to other Anaeromyxobacter species:

0 As cytochrome (and other metal-reduction) deficient mutants of 2CP-C become available, we will conduct a series of phenotype characterization experiments. These will include: a) reduction kinetics of different forms of Fe (III) (HFO, Fe(III)- citrate/NTA), Mn(IV), U(VI), and Tc(VII) and b) growth on and reduction of other e-acceptors; c) high-resolution transmission electron microscope image analysis.

o We will also carry out comparative experiments with other sequenced strains of Anaeromyxobacter (2CP-C, 2CP-1, strain K, strain FW-109) targeting reduction of various metals and radionuclides.

- Elucidation of the global transcriptome profiles of A. dehalogenans 2CP-C under different respiratory conditions

o Identification of baseline expression profiles in 2CP-C cultures growing under different single-electron acceptor respiratory conditions (HFO, Fe(III)-NTA, fumarate, nitrate, and 2-chloro phenol).

o Expression studies will be also conducted under controlled conditions with respect to growth rate, electron donor and acceptor concentrations, and relevant 
parameters such as dissolved oxygen, temperature and $\mathrm{pH}$. Upon achieving steady state growth conditions, the cells from continuous cultures will be transitioned to electron donor-limiting and non-limiting conditions using various concentrations of metal-electron acceptors

\section{Information Access:}

\section{$\underline{\text { Publication }}$}

Wu, Q., Sanford, R.A., and Loffler, F.E. (2006) Uranium(VI) reduction by Anaeromyxobacter dehalogenans strain 2CP-C. Appl Environ Microbiol 72: 3608-3614.

\section{$\underline{\text { Recent Presentations \& Meeting Abstracts }}$}

Beliaev, A.S., F. E. Loeffler, R. A. Sanford, and J. K. Fredrickson. 2006. Biomolecular Mechanisms Controlling Metal and Radionuclide Transformations in Anaeromyxobacter dehalogenans. Abstract submitted to Environmental Remediation Sciences program (ERSP) 1st Annual PI Meeting, Warrenton, VA.

Marshall, M. J., A. S. Beliaev, D. W. Kennedy, A. Dohnalkova, A. E. Plymale, S. H. Thomas, F. E. Loeffler, R. Sanford, S. B. Reed, D. E. Culley, Y. Zhang, D. Saffarini, M. F. Romine, J. M. Zachara, and J. K. Fredrickson. 2006. Biochemical Mechanisms of Technetium(VII) Reduction by Shewanella oneidensis and Anaeromyxobacter dehalogens. Abstract submitted to 11th International Symposium on Microbial Ecology (ISME-11), Vienna, Austria.

Thomas, S.H., M. Pavlekovic, N. Lee, M. J. Marshall, D. W. Kennedy, K. M. Ritalahti, R. A. Sanford, and F. E. Loeffler. 2006. Diversification in the Subsurface: Strain Variation of Anaeromyxobacter dehalogenans in a Uranium and Nitrate-Contaminated Subsurface Environment. Abstract submitted to the 11th International Symposium on Microbial Ecology (ISME-11), Vienna, Austria. 

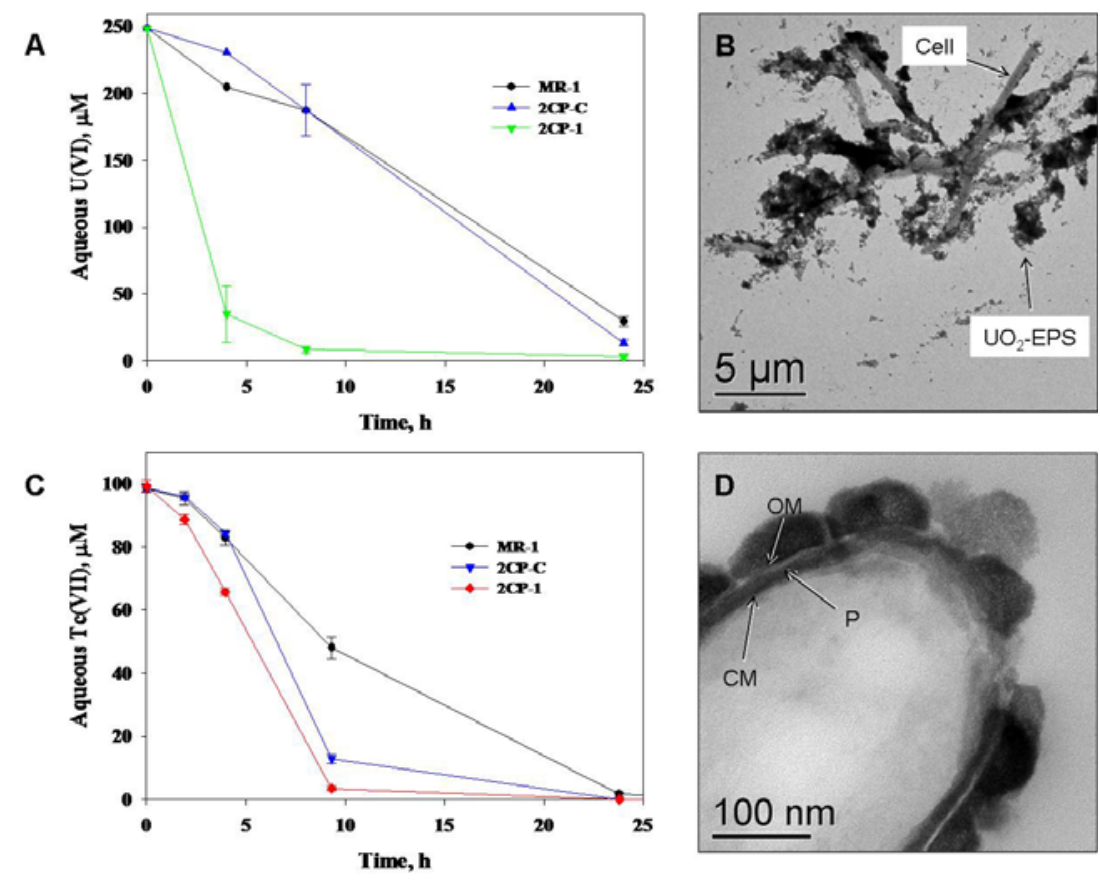

Figure 1. Summary of radionuclide reduction and localization in A. dehalogenans strains 2CP-C and 2CP-1. Reduction kinetics was determined from standardized resting cell suspensions incubated with either uranyl acetate (A) or ammonium pertechnetate (C) and hydrogen. Whole mount transmission electron micrographs reveal the extracellular localization of $\mathrm{UO}_{2}$-EPS (B) in association with intact 2CP-C cells. Thin sections prepared from $2 \mathrm{CP}-1$ cells show the multiple sub-cellular locations of $\mathrm{TcO}_{2}$ nanoparticles relative to the cell membrane (CM), periplasm (P), and outer membrane (OM).

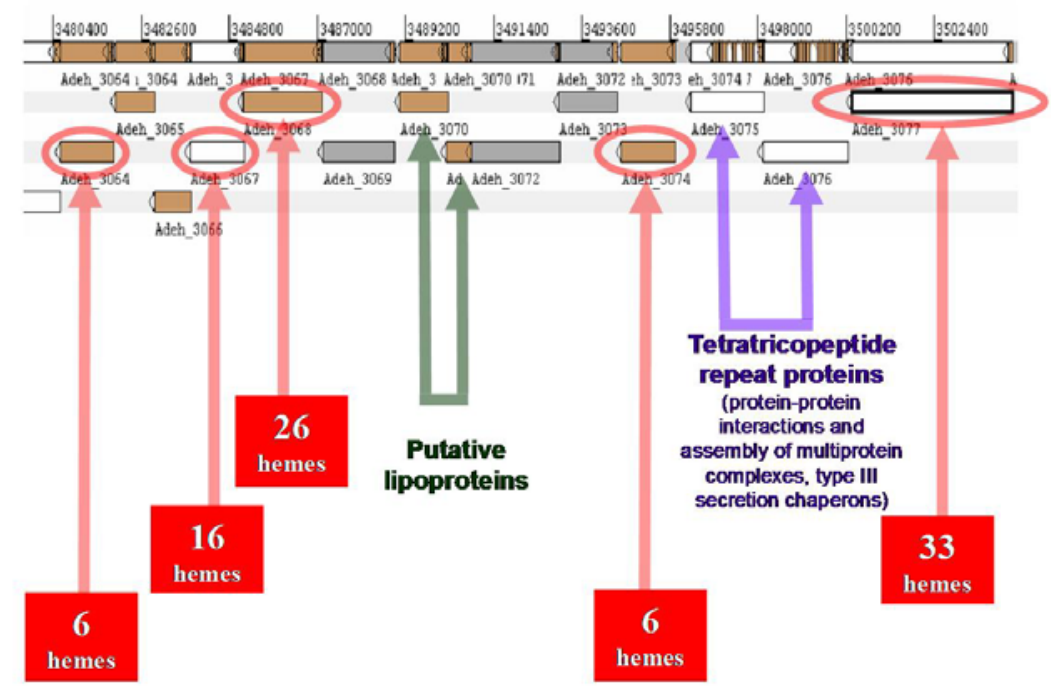

Figure 2. A major 40-kb multi-cytochrome gene cluster was identified within the $A$. dehalogenans 2CP-C genome. The organization of the five putative $c$-type cytochromes and the predicted number of $c$-type heme-binding domains in each protein are shown. 